\title{
Fabrication of Nanoporous Al by Vapor-Phase Dealloying: Morphology Features, Mechanical Properties and Model Predictions
}

\author{
Andrea Pinna ${ }^{1}(\mathbb{D})$, Giorgio Pia ${ }^{1,2,3, *}$, Maria Francesca Casula ${ }^{1}$, Francesco Delogu ${ }^{1,2,3}$, Elisa Sogne ${ }^{4}$ (D), \\ Andrea Falqui 4,5 and Luca Pilia $1, *$ (D)
}

1 Dipartimento di Ingegneria Meccanica, Chimica e dei Materiali, Università degli Studi di Cagliari, Piazza d'Armi, 09123 Cagliari, Italy; andreapinna27@gmail.com (A.P.); casulaf@unica.it (M.F.C.); francesco.delogu@dimcm.unica.it (F.D.)

2 Consorzio Sistemi a Grande Interfase (CSGI), Department of Chemistry, University of Florence, via della Lastruccia 3, 50019 Sesto Fiorentino, Italy

3 Cagliari Research Unit, via Marengo 2, 09123 Cagliari, Italy

4 NABLA Lab, Biological and Environmental Sciences and Engineering (BESE) Division, King Abdullah University of Science and Technology (KAUST), Thuwal 23955-6900, Saudi Arabia; eli.sogne@gmail.com (E.S.); andrea.falqui@unimi.it (A.F.)

5 Dipartimento di Fisica, Università degli Studi di Milano, via Celoria 16, 20131 Milano, Italy

* Correspondence: giorgio.pia@dimcm.unica.it (G.P.); pilialuc@unica.it (L.P.); Tel.: +39-706-755-051 (G.P.); +39-706-755-063 (L.P.)

Citation: Pinna, A.; Pia, G.; Casula, M.F.; Delogu, F.; Sogne, E.; Falqui, A.; Pilia, L. Fabrication of Nanoporous Al by Vapor-Phase Dealloying: Morphology Features, Mechanical Properties and Model Predictions. Appl. Sci. 2021, 11, 6639. https:// doi.org/10.3390/app11146639

Academic Editor: Karol Ćwieka

Received: 29 June 2021

Accepted: 16 July 2021

Published: 20 July 2021

Publisher's Note: MDPI stays neutral with regard to jurisdictional claims in published maps and institutional affiliations.

Copyright: (c) 2021 by the authors. Licensee MDPI, Basel, Switzerland. This article is an open access article distributed under the terms and conditions of the Creative Commons Attribution (CC BY) license (https:// creativecommons.org/licenses/by/ $4.0 /)$.

\begin{abstract}
The physical and chemical properties shown by nanoporous metals, related to their unique structure, make them very promising for application in several fields. Recently, vapor-phase dealloying has been reported as a method for the preparation of several non-noble nanoporous metals, alternatively to dealloying in aqueous solutions. Using this approach, we have successfully fabricated nanoporous $\mathrm{Al}$ starting from an $\mathrm{Al}_{20} \mathrm{Zn}_{80}$ nanocomposite obtained by ball milling. The nanocomposite was annealed at $550{ }^{\circ} \mathrm{C}$ under high-vacuum conditions, and the difference in the vapor pressures allowed the selective removal of $\mathrm{Zn}$ by vapor-phase dealloying. The morphology of the resulting nanoporous material was analyzed by Scanning Electron Microscopy showing pores from few to thousands of $\mathrm{nm}$; moreover, the nanoporous 3D structure was observed through Serial Block Face-Scanning Electron Microscopy. A specific surface area as high as $73 \mathrm{~m}^{2} \mathrm{~g}^{-1}$ was estimated by $\mathrm{N}_{2}$ physisorption measurements. In addition, a fractal model able to well reproduce the morphology of nanoporous $\mathrm{Al}$ was built. This model has been used for predicting mechanical properties which are in good agreement with experimental data obtained by nanoindentation.
\end{abstract}

Keywords: nanoporous metals; nanocomposites; aluminum; dealloying; mechanical properties; fractal model

\section{Introduction}

Porous materials can be simply defined as materials in which a fraction of the solid is replaced by pores [1]. Depending on their open or closed form, they exist as isolated cavities, ordered channels or percolating networks of passages [1]. The relative volume fraction occupied by pores and their distribution inside the matrix determine, to various extents, several properties of the porous material [2]. Among this class of materials, porous metals in both forms, metal foams [3] and nanoporous (NP) [4], which differ due to their different morphology and pore sizes, are extensively studied because of their potential applications in several technological fields [4,5].

The combination of high stiffness with very low specific weight, high compression strengths and good energy absorption characteristics made metal foams attract interest from companies and industries, which finally led to a significant expansion of knowledge on manufacturing and possible applications [3]. Both closed- and open-cell metal foams 
have a wide spectrum of potential applications, ranging from the automotive and aerospace industry to shipbuilding and civil engineering [5]. The investigation of structure-property relationships appeared immediately crucial for interpreting experimental findings and tailoring the foam mechanical properties to specific applications.

NP metals exhibit physical and chemical properties that promise application in several science and engineering areas [6]. The entire set of properties stems from the unique morphology of NP metals characterized by a disordered and intricate structure with a remarkable specific surface [7-9]. NP metals offer great potential as structural materials. They combine, indeed, the properties and workability of bulk metals with a much lower density. In addition, scale effects that originate for characteristic lengths in the nanometer range could result in stronger NP metal foams than bulk metal counterparts by at least one order of magnitude [10].

NP metals are typically obtained by dealloying, i.e., by the selective removal of the less noble constituents from the precursory alloy [11]. Although, with the due shrewdness, noble metals can be readily fabricated in NP form by chemical etching in an aqueous solution, possibly assisted by electrochemical forcing. NP Au is the most studied NP metal, thanks to the solid solubility with $\mathrm{Ag}$ and its low reactivity. Its bicontinuous nanoporous structure confers to NP Au better mechanical properties than corresponding bulk metals; however, its high costs hinder the use of this material for structural applications.

In this regard, the ability to fabricate other metals in NP form is crucial in order to facilitate their access to commercial applications, such as structural ones. Nevertheless, the fabrication of NP structures remains challenging for most base metals because of their propensity to oxidation. In order to overcome this drawback, different fabrication methods have been reported. For example, some of them (such as $\mathrm{Ti}, \mathrm{Ta}, \mathrm{Si}, \mathrm{Nb}$ ) have been obtained replacing the aqueous solution with a liquid metal [12-14]. In this respect, vapor-phase dealloying (VPD) has been recently developed [15]. This method takes advantage of the differences in the vapor pressures of elemental metals to selectively remove one or more alloy constituents, regardless of the propensity to oxidation.

Among metals, aluminum shows important applications in transportation and construction because of its properties such as plasticity, a lightweight nature and passivation capability. In this regard, NP Al fabrication represents an essential goal for possible and innovative further applications in relevant advanced fields. Indeed, recently in the literature, it has been shown that $\mathrm{NP} \mathrm{Al}$ is a promising material for hydrogen generation [16] and ultraviolet (UV) plasmonic applications, such as Surface Enhanced Raman Spectroscopy (SERS) and Metal Enhanced Fluorescence (MEF) [17]. Plasmon resonance of $\mathrm{Al}$ nanostructures can be tuned by varying their size, structure, and oxide content from UV to near-infrared (NIR) $[18,19]$. These features make this material a promising valuable low-cost, self-supported, and tunable substrate for UV and visible-light SERS and MEF. Moreover, NP Al could also be of interest as a catalyst by matching the unique surface area and catalytic properties of NP metals with the Al catalytic activity for various organic reactions [20]. Furthermore, the use of self-standing nanostructured material such as a NP metal for catalytic purposes could allow taking the advantages of both homogeneous and heterogeneous catalysis without the pelletizing process, which can be required when nanoparticles are employed as catalysts [21,22].

$\mathrm{NP} \mathrm{Al}$ fabrication is challenging because of the high $\mathrm{Al}$ reactivity toward oxygen and water. The first of the few papers illustrating the preparation of a NP Al has been reported by Suárez et al.; it describes a fabrication method based on the electrochemical dealloying of $\mathrm{Zn}$ or $\mathrm{Mg}$ [23]. Later, Corsi et al. [16] demonstrated that NP Al could be prepared by this method in non-aqueous electrolytes under an Ar atmosphere, thus preventing both $\mathrm{Al}$ oxidation during its removal and the ligament coarsening observed by Yang et al. [24] during Galvanic Replacement Reaction.

In this work, we address the production of NP aluminum by VPD by treating an $\mathrm{Al}_{20} \mathrm{Zn}_{80}$ nanocomposite at a relatively high temperature under a vacuum. Three steps are involved in the fabrication method: mechanical alloying of $\mathrm{Al}$ and $\mathrm{Zn}$ powders under an 
Ar atmosphere, cold-pressing of powders into pellets, and $\mathrm{Zn}$ removal during a thermal treatment under vacuum conditions. Although ligament coarsening could occur because of the relatively high temperature required with VPD, this preparation method is promising because of its ease and the possibility of fabricating relatively large amounts of materials.

Structure features have been investigated at different magnifications by Scanning Electron Microscopy (SEM) and Serial Block Face-Scanning Electron Microscopy (SBF-SEM), while surface area and pore volume have been characterized by $\mathrm{N}_{2}$ physisorption measurements.

Since mechanical properties of NP Al are not studied in an exhaustive way, nanoindentation tests have been performed to estimate Young's modulus for the first time.

A further investigation of microstructure morphology has been performed by using a phenomenological model based on fractal geometry. Moreover, relevant applications of fractal modeling show its reliability not only to reproduce structure features of several materials, but also to predict their behavior under different conditions [25-27]. In this case, it has been possible to calculate the specific surface (by simple geometrical measurements) and Young's modulus (by resolving series and parallel springs' patterns after model conversion), which have then been successfully compared with experimental measurements.

\section{Materials and Methods}

\subsection{NP Preparation}

Experiments were carried out using granular $\mathrm{Al}$ with a particle size of $<1 \mathrm{~mm}$ and $99.7 \%$ purity, and Zn powders with a particle size of $<0.149 \mathrm{~mm}$ and $99.8 \%$ purity. Elemental metals were mixed to prepare $\mathrm{Al}_{20} \mathrm{Zn}_{80}$ powder mixtures. The corresponding $\mathrm{Al}_{20} \mathrm{Zn}_{80}$ alloy was obtained by subjecting the powder mixtures to mechanical processing in a SPEX Mixer/Mill 8000 (SPEX SamplePrep, LLC, Metuchen, NJ, USA). To this aim, $10 \mathrm{~g}$ of powder mixture were placed inside a cylindrical hardened steel reactor together with two $8 \mathrm{~g}$ stainless steel balls. Paraffin oil was used as a process-control agent to avoid powder agglomeration. The reactor was sealed under an Ar atmosphere with impurities below $2 \mathrm{ppm}$. The mechanical processing was interrupted after $48 \mathrm{~h}$. The structural and microstructural evolution of processed powders was investigated by X-ray Diffraction (XRD). XRD measurements were carried out with a Rigaku Miniflex II Diffractometer (Rigaku, Tokyo, Japan) equipped with a $600 \mathrm{~W}$ X-ray source using $\mathrm{Cu} \mathrm{K}_{\alpha 1}$, with an incident beam angle $2 \theta$ ranging from 30 to 100 degrees. Quantitative phase and microstructural analyses were performed using the Rietveld method [28]. Processed powders were coldpressed in the form of cylindrical pellets about $1.3 \mathrm{~cm}$ in diameter and $0.3 \mathrm{~mm}$ in thickness. The pellets were placed in the glass tube of a tubular furnace. The sample was kept for $1 \mathrm{~h}$ under high-vacuum conditions with pressure around $10^{-2} \mathrm{~Pa}$. Then, the temperature was raised at $25^{\circ} \mathrm{C} \mathrm{min}-1$ up to $550{ }^{\circ} \mathrm{C}$. The sample was annealed at $550{ }^{\circ} \mathrm{C}$ for $1 \mathrm{~h}$ and then cooled gradually under high-vacuum conditions.

\subsection{SEM Characterization}

The resulting material was investigated by SEM using a Zeiss (Oberkochen, Germany) Merlin microscope equipped with a Schottky electron source, working with an acceleration voltage of $5 \mathrm{kV}$ and an electron current of $150 \mathrm{pA}$ at a short working distance (highresolution mode). Secondary electrons (SE) were collected to provide fine details on the surface morphology, while backscattered electrons (BSE) were collected to detect differences in local composition. SE and BSE were gathered using an in-lens detector and a multiplesector detector placed on the bottom of the SEM objective lens, respectively. SEM-Energy Dispersive Spectroscopy (EDS) measurements were performed with an Oxford silicon drift detector (SDD) (Oxford Instruments, Abingdon, UK) with a detection area of $60 \mathrm{~mm}^{2}$, collecting EDS spectra from 6 diverse, rectangular-shaped cross-sectional areas placed at increasing depths in the sample, aiming at finally covering the entire $224 \mu \mathrm{m}$-thick sample. In order to properly compare the results obtained, rectangular zones with the same surface area were scanned by the electron beam, and an EDS spectrum from each of them was 
collected keeping the same SEM working conditions: acceleration voltage $(30 \mathrm{kV})$, electron probe current ( $1 \mathrm{nA})$, and collection real-time ( $5 \mathrm{~min}$ per spectrum).

SEM images were subjected to image analysis (IA) using AutoCad ${ }^{\circledR}$ (Version 24.0) to identify pores. To account for their irregular shapes, different characteristic lengths were roughly estimated per each pore, and average pore size was eventually calculated.

\section{SBF-SEM Imaging}

For SBF-SEM imaging, the specimen was first embedded in EM-dedicated epoxy resin to obtain a small and compact block, then such a block was mounted on an Al specimen. Silver paint was used to electrically ground as much as possible the edges of the block to the aluminum pin. The entire block was then sputter-coated with a layer of gold capable of reflecting light to perform the fine SBF-SEM alignment. The block containing NP Al was then imaged using a ThermoFischer (Waltham, MA, USA) VolumeScope (VS) device, mounted into a ThermoFischer Teneo FEG SEM equipped with a Schottky field emission electron source, operating at an accelerating voltage of $2 \mathrm{kV}$, with a beam current of $50 \mathrm{pA}$, and under low-vacuum conditions (50 Pa). Serial BSE-SEM images were acquired by a dedicated detector after cutting the specimen with a thickness of $100 \mathrm{~nm}$ at different magnifications, and with resolution and dwell times chosen adequately to prevent further local specimen charging. For 3D reconstruction, rendering, and analysis, serial SEM images were aligned and stacked into a volume using AVIZO software (version 2020.3) (ThermoFischer). Three-dimensional structures in image stacks containing hundreds or thousands of 2D ortho slices were traced individually in each plane and automatically surface rendered. From SBF-SEM stacks analysis, the features of larger pores (such as area, shape, characteristic lengths, and pore profile roughness) were estimated through a semi-automatic tool (Analyze Particles) using Fiji program (version 1.53c plus) [29]. Fractal dimension was estimated by using "Fractal Box Count" tool of Fiji [29].

\section{3. $\mathrm{N}_{2}$ Physisorption Measurements}

$\mathrm{N}_{2}$ physisorption measurements were carried out using a Micromeritics (Norcross, GA, USA) ASAP2020 apparatus. Adsorption and desorption isotherms were measured at $77 \mathrm{~K}$. The Brunauer-Emmett-Teller (BET) method was utilized to estimate the specific surface area [30].

\subsection{Mechanical Properties Measurements}

The mechanical properties of suitably prepared NP Al samples were measured at room temperature using ex situ depth-sensing nanoindentation. To this aim, a NanoTest Vantage Micro Materials indenter (Micro Materials, Wrexham, UK), equipped with a calibrated three-sided pyramid diamond Berkovich tip about $200 \mathrm{~nm}$ in radius, was used. Measurements were carried out within the indentation load range between 400 and $2400 \mu \mathrm{N}$. The loading rate was kept constant at $500 \mu \mathrm{N} \mathrm{s}^{-1}$. Loading and unloading curves were measured on 36 indents per sample. The slope of the linear part of each unloading curve provides an estimate of the Young's modulus. The average Young's modulus was calculated by taking into account all the measured values.

\subsection{Fractal Model}

A phenomenological model based on fractal geometry was developed with the aim to reproduce the porous structural features (pore size distribution, pore size range, pore fraction) and predict the specific surface area and Young's modulus of NP Al [25]. In particular, the proposed fractal model, called IFU, was obtained by using Sierpinski carpet, a well-known example of fractal geometry as a base unit. This fractal figure was constructed by starting from a square in which each side $(l)$ is divided by a numerical factor $F$ generating 
equal sub-squares (Equation (1)). Some of these sub-squares can be removed by creating a structure formed by solids and voids with the same characteristic length [25].

$$
L_{e}^{(\max )}=\frac{l}{F}
$$

The obtained objects were characterized by the non-integer dimension calculated by

$$
D_{f}=\frac{\log \left(N_{R S Q}\right)}{\log (F)}
$$

where $N_{R S Q}$ denotes the number of sub-squares not removed at the first iteration. The described step was repeated on all sub-squares, generating a self-similar pattern in which at the $n$-th iteration, the size of the voids was:

$$
L_{e}^{(n)}=\frac{L_{e}^{(\max )}}{F^{(n-1)}}
$$

Iteration by iteration, it was possible to note that new classes of voids were generated, and monotonic pore size distribution could be obtained. However, real materials in general have different pore size distribution curves (non-monotonic) in which one or more maxima can be found in correspondence with any pore class. These structures could be approximated by a modeling procedure based on IFU. The number of unit base type was related to the complexity of the cumulative curve of porous structures, one unit for every inflection point. For the investigated systems, two structural types of units' bases were used: 1-unit Sierpinski carpet $A$ and $n_{B}$ Sierpinski carpet B (for simplicity unit $\mathrm{A}$ and unit B), with different characteristic lengths and $D_{f}$.

The pore fraction resulting from intermingling was given by:

$$
\varepsilon_{\text {mod }}=\left(A_{A p}+n_{B} \cdot A_{B p}\right) /\left(A_{A}+n_{B} \cdot A_{B}\right)
$$

where $A_{A}, A_{B}, A_{A p}$, and $A_{B p}$ are the total areas and total pore areas of $\mathrm{A}$ and $\mathrm{B}$ units, respectively. After porosimetric tests or image analysis, it was possible to use the experimental measurement value for calculating $n_{B}$

$$
n_{B}=\left(A_{A p}-\varepsilon_{\exp } \cdot A_{A}\right) /\left(\varepsilon_{\exp } \cdot A_{B}-A_{B p}\right),
$$

where $\varepsilon_{\text {exp }}$ is the experimental pore fraction. In order to improve the versatility of the model, different settings could be used, such as: (a) maintain solid some sub-squares of unit A and units B forever; (b) introduce non-porous surfaces to control the pore fraction of the entire model; (c) make the pore profile tortuous in a longitudinal or transverse direction for reproducing specific surface and morphological characteristics. In particular, concerning the tortuous pore profile, it can be characterized by rough path $L_{t}^{(n)}$ which was calculated by

$$
L_{t}^{(n)}=L_{e}^{(n)} \delta^{-i^{1-D_{t}}}
$$

where $D_{t}$ is tortuous fractal dimension and

$$
\delta=F^{-n}
$$

A tortuous pore profile was constructed around the square edge of a void to leave the solid and void fraction unaffected. Consequently, the pore cross-sectional area was considered constant and independent of the specific profile. Since the IFU is the smallest representative part of a microstructure, a method to compare data with IA (referred to the 
examined surface) had to be used. For this, the total length of pore profiles was divided by total model surface $\left(A_{I F U}\right)$,

$$
L_{S}=\frac{\sum_{1}^{n} L_{e}^{(n)} \delta^{-i^{1-D_{t}}}}{A_{I F U}}
$$

Moreover, it was possible to calculate specific surface area by considering IFU unitary model extrusion along the transversal direction and the specific weight of material solid phase, $w_{s}$ :

$$
S_{s}=\frac{L_{s} t}{w_{s}}
$$

After phenomenological reconstruction of the microstructure, the fractal model can be converted into an analytical multi-scale homogenization pattern composed of springs allocated in series and parallel. Starting from the smallest cell (generated by the last iteration $n)$, the effective Young's modulus $E^{(i)}$ was computed, and the result was used as the modulus of a solid part at $n-1$ iteration [25].

At the $n$ iteration, an analytical expression for stiffness of each spring was represented by

$$
k_{q r}^{(n)}=\chi_{q r} E_{s} \frac{A_{e}^{(n)}}{L_{e}^{(n)}}, \quad q=I, I I, I I I, \quad r=1,2,3,
$$

where $\chi_{q r}$ is a characteristic function which is equal to 0 for pore and 1 for solid, $E_{s}$ denotes the elastic modulus of the solid phase, $A_{e}^{(n)}$ represents the cross-sectional area (per unit out-of-plane thickness), and $L_{e}^{(n)}$ stands for the length of the corresponding sub-square. These last geometrical features were the same in all sub-squares. The effective Young's modulus was calculated by

$$
E^{(n)}=\left[\sum_{q=I, I I, I I I}\left(\frac{1}{\sum_{r=1,2,3} E_{q r}^{(n)}}\right)\right]^{-1},
$$

where $E_{q r}^{(n)}=\chi_{q r} E_{s}[25]$.

The Young's modulus of the entire fractal structure was given by the harmonic average of the Young's modulus of each base unit and filled surface. The three-dimensional structure was reproduced by giving an arbitrary unit thickness to the IFU.

\section{Results and Discussion}

The XRD pattern of the $\mathrm{Al}_{20} \mathrm{Zn}_{80}$ powder mixture mechanically processed for $48 \mathrm{~h}$ is shown in Figure 1a and compared with the reference XRD patterns of pure $\mathrm{Al}$ and $\mathrm{Zn}$. The mechanical processing made $\mathrm{Al}$ reflections disappear and $\mathrm{Zn}$ ones broaden. The Rietveld analysis indicates that broadening can be ascribed to the reduction of grain size from the initial value of about $100 \mathrm{~nm}$ to the final one of about $70 \mathrm{~nm}$. A small downward shift of about $0.1^{\circ}$ in the angular position of $\mathrm{Zn}$ peaks is detected. Based on the $\mathrm{Al}$ and $\mathrm{Zn}$ atomic radii [31], it can be ascribed to a terminal solid solubility of $\mathrm{Al}$ in $\mathrm{Zn} \mathrm{[32],} \mathrm{with} \mathrm{the} \mathrm{two}$ elements remaining substantially segregated. 


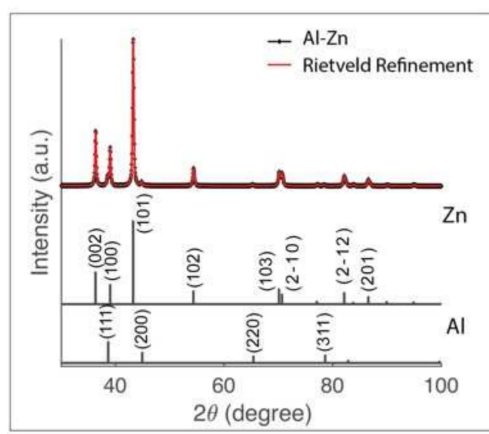

(a)

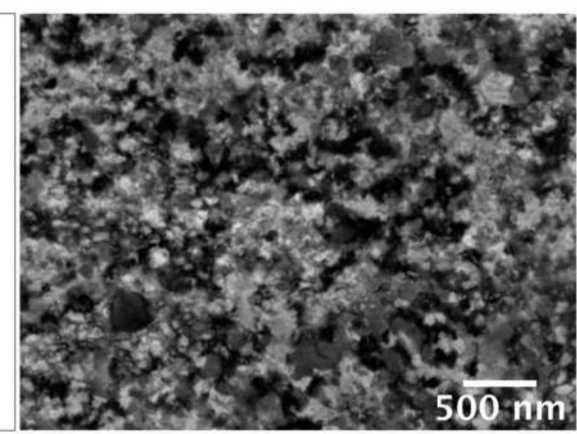

(b)

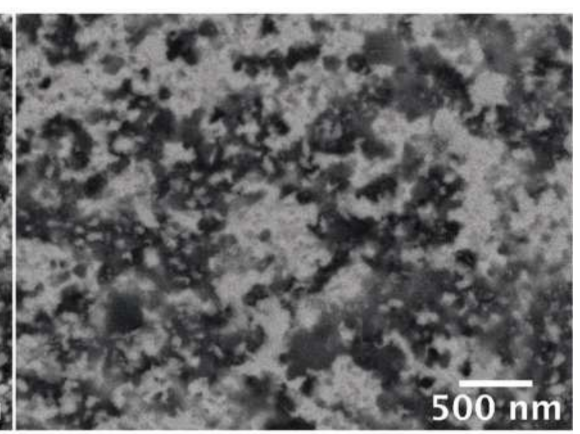

(c)

Figure 1. (a) XRD pattern and Rietveld refinement of the $\mathrm{Al}_{20} \mathrm{Zn}_{80}$ nanocomposite, compared with reference reflection peaks of $\mathrm{Al}$ and $\mathrm{Zn}$ elements. SEM micrographs of the nanocomposite collected using (b) SE and (c) BSE.

Therefore, it can be concluded that the mechanical processing of the $\mathrm{Al}_{20} \mathrm{Zn}_{80}$ powder mixtures induces the formation of a nanocomposite. The SEM micrographs, such as the ones shown in Figure 1b,c, provide support for this conclusion. In particular, the SEM-BSE imaging indicates that the nanocomposite consists of finely dispersed nanocrystalline phases with diverse atomic numbers. Specifically, the zones appearing brighter have a higher atomic number, i.e., a higher Zn percentage. As shown by the SEM micrographs reported in Figure 2, the annealing at $550{ }^{\circ} \mathrm{C}$ under high-vacuum conditions for $1 \mathrm{~h}$ drastically changes the material microstructure. As shown in Figure 2a, the sample surface exhibits the typical appearance of NP metals with nanosized ligaments and pores (approximately $10 \mathrm{~nm}$ ). The SEM micrograph in Figure $2 \mathrm{~b}$ reveals that the NP architecture is maintained inside the sample, although the ligament size appears to be larger.

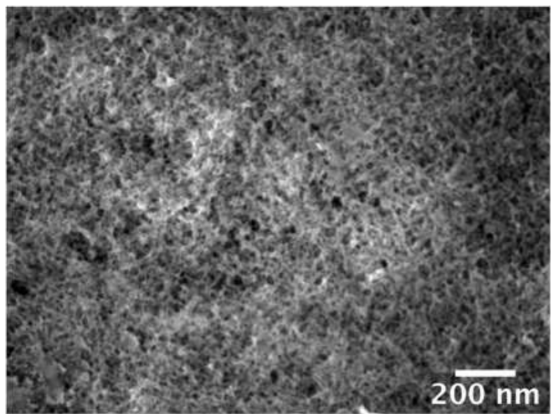

(a)

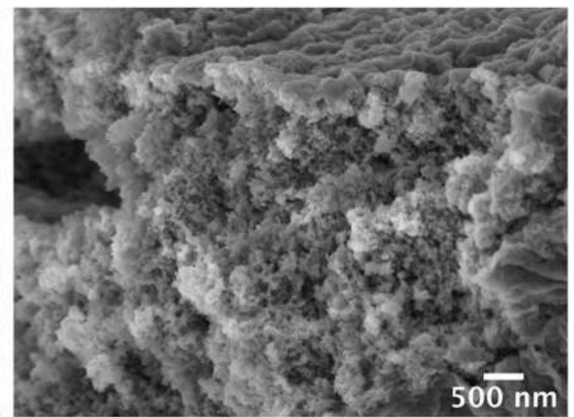

(b)

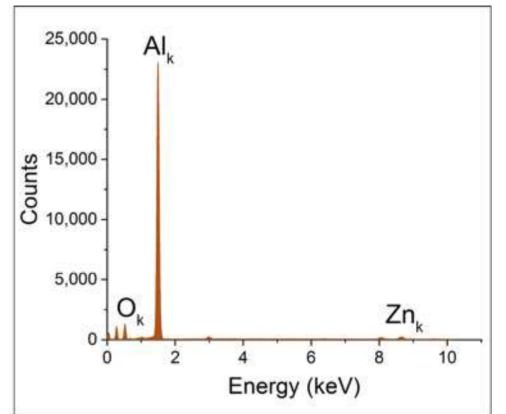

(c)

Figure 2. SEM-SE micrographs of (a) the NP Al surface and (b) the NP Al cross-section; (c) EDS spectrum corresponding to the cross-sectional area included between 37 and $74 \mu \mathrm{m}$ from the pellet surface with $\sim 3 \%$ of $\mathrm{Zn}$ content. The spectra of the other areas of the sample are reported in Figure S1.

EDS measurements shown in Figure 2c and Figure S1 indicate that the Zn atomic percentage, normalized with respect to the sum of the $\mathrm{Al}$ and $\mathrm{Zn}$ ones, mostly averages around $1 \%$ near the pellet surface, while it increases up to about $3 \%$ near the opposite surface. Therefore, $\mathrm{Zn}$ has been almost completely removed from the sample. The $\mathrm{O}$ content is related to the oxidation of the Al surface, with consequent formation of a thin passivation layer [33].

The experimental evidence heretofore presented definitely suggests that we have been able to fabricate almost pure NP Al. The architecture we observe can be well described in terms of open cells with irregular morphologies that tessellate the entire sample volume. Therefore, the NP Al can be considered a truly NP metal with ligaments characterized by a relatively high aspect ratio, which gives them their stocky appearance. It is possible to note that the sample shows a porous structure over its whole volume. However, relatively 
large voids, such as the one shown in Figure 3a, have been observed. Our first hypothesis is that they are due to the incomplete compaction of the nanocomposite powders during the preparation of pellets.

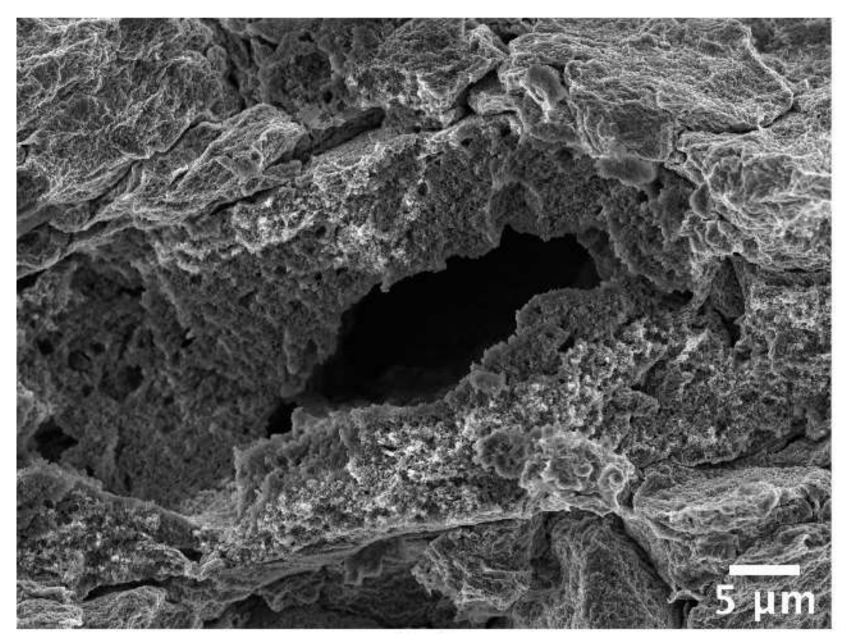

(a)

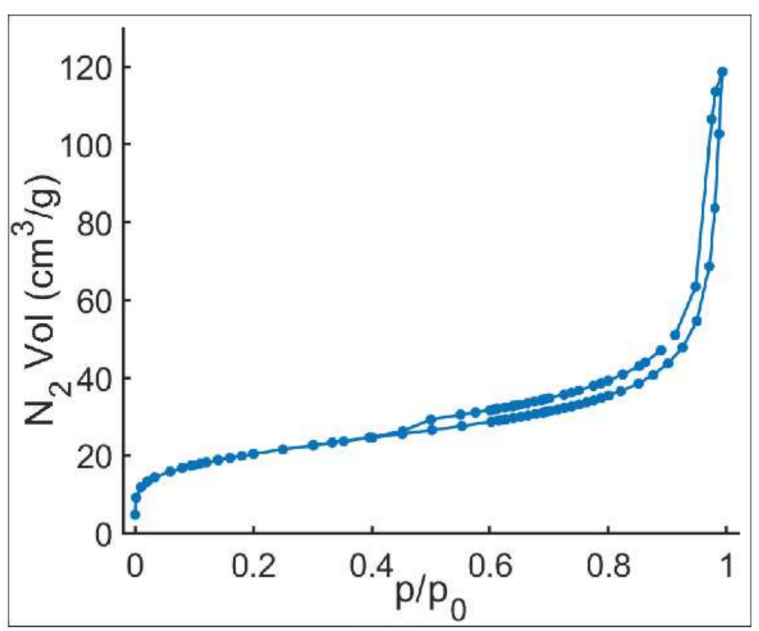

(b)

Figure 3. (a) SEM-SE micrograph of the NP Al cross-section showing a quite large internal pore. (b) $\mathrm{N}_{2}$ physisorption isotherms obtained from the whole NP Al specimen.

The shape of the $\mathrm{N}_{2}$ physisorption isotherms shown in Figure $3 \mathrm{~b}$ is associated with the occurrence of a hysteresis loop and does not strictly match with a specific classification, supporting the NP Al structure's inhomogeneity [34]. The NP Al specific surface area, as obtained by BET analysis, is nearly $73 \mathrm{~m}^{2} \mathrm{~g}^{-1}$, a remarkably large value that compares well with the highest literature values for NP metals $[35,36]$. Although limitations associated to surface area determination by physisorption routes have been reported, the correlation with the structural features has been pointed out [35-37]. For instance, NP Au can have a specific surface area as large as $85 \mathrm{~m}^{2} \mathrm{~g}^{-1}$ when its ligaments are about $7 \mathrm{~nm}$ in size [35]. From pore volume evaluation, a porosity of $40 \%$ can be estimated, relative to those pores smaller than $300 \mathrm{~nm}$.

The presence of nanosized ligaments and pores represents a fundamental aspect of a NP materials. However, it is well known that the performances of this kind of material also depend on larger-scale cavities by influencing various parameters, such as the mass transport of fluids, mechanical properties, and electrical and thermal conductivity [38,39]. Thus, aiming at investigating the overall porous structure of the specimen, a technique capable of revealing its internal 3D structure appeared of pivotal importance. As a consequence, an SBF-SEM experiment was carried out on it. By this technique, the sample 3D structure along the entire $270 \mu \mathrm{m}$ cross-section profile was determined (example images of the cross-section at two different magnifications are shown in the Supplementary Materials, Figure S2) and the obtained micrographs allow analysis of NP Al at different scales with a resolution of $300 \mathrm{~nm}$.

From the SBF-SEM measurements, the 3D reconstruction and a representative video of the porous structure evolution during the subsequent cuts of the entire cross-section profile are shown in Figure 4 and in Video S1, respectively. Quantitative analysis of SBF images was focused near the surfaces and in the interior of the sample (see Figure S3). Indeed, it can be observed that the two surfaces have smaller pores and a higher overall porosity with respect to the inner part of the sample, as can also be clearly seen by the 3D reconstructions at a low (Figure 4) and higher (Figure S4) magnification. Moreover, the dense areas of the sample manifest different brightness levels, associated with a higher concentration of $\mathrm{Zn}$ in the brightest areas (and a lower one in the inverted images in Figures S2 and S3). The 
$\mathrm{Zn}$-richest areas are found to be more prevalent in the interior of the specimen due to the slower $\mathrm{Zn}$ removal in the inner part of the sample.

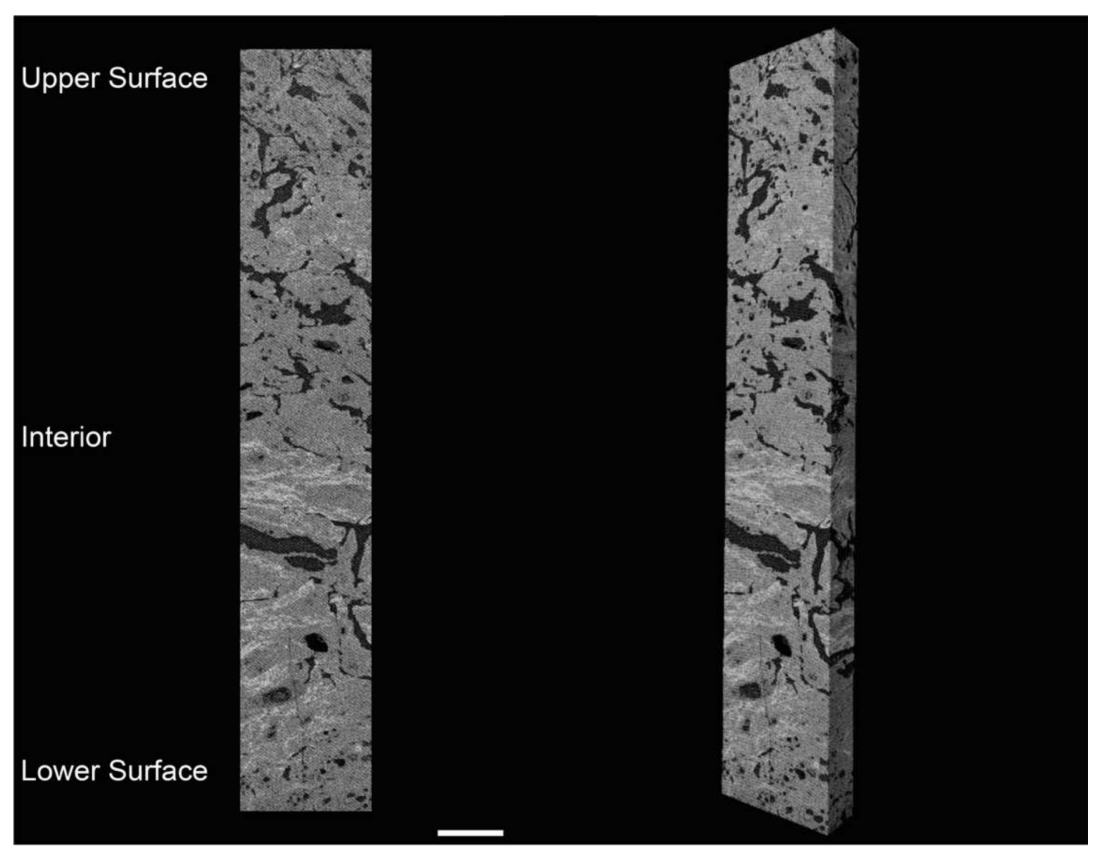

Figure 4. SEM-SBF 3D reconstruction of cross-section profile of NP Al; scale bar: $20 \mu \mathrm{m}$.

From SBF-SEM analysis and by using IA techniques, it has been possible to estimate an additional porosity of $10 \%$ related to pores larger than $300 \mathrm{~nm}$, which cannot be estimated by $\mathrm{N}_{2}$ physisorption measurements. Therefore, by adding this value to the porosity relative to pores smaller than $300 \mathrm{~nm}$, a rough estimation of the overall porosity lies at around $50 \%$. IA of SBF-SEM images was also carried out to elaborate pore cumulative curves for voids with characteristic lengths higher than $300 \mathrm{~nm}$. Consequently, in order to reproduce these experimental findings, an IFU model (see Figure 5) has been applied and input data has been reported in Table 1. In Figure 6a, a cumulative curve obtained by IA elaborations has been compared with fractal pore structure. It is possible to observe that the proposed model can reproduce the experimental data. Moreover, IA allows calculating the fractal dimension (Dt) of pores that is about $1.17 \pm 0.02$. By using Equation (8), it is possible to estimate the length of the tortuous profile of each pore. Furthermore, in this case, the measured and calculated data, referred per unit of surface, are very comparable, $0.33 \mu \mathrm{m}^{-1}$ and $0.30 \mu \mathrm{m}^{-1}$, respectively. However, the pore fraction relative to IA observation is only $1 / 5$ of the total porosity (equal to $50 \%$ ). In order to have an overall description of porosity, IFU models have been used to design possible pore size distributions. These designed cumulative curves are built considering pore size ranges from SEM observations and overall porosity from SBF-SEM stacks and $\mathrm{N}_{2}$ physisorption measurements. In Figure $6 \mathrm{~b}$, pore size distributions designed by using input data reported in Table 2 have been shown. It is possible to notice that, as supposed, pore size range and pore fraction are equal for every cumulative curve, yet they have different pore size distributions. The remarkable capability of the model to reproduce experimental data is confirmed by the calculated specific surface (Equation (8)), which is equal to $73 \pm 7 \mathrm{~m}^{2} / \mathrm{g}$ vs. about $73 \pm 1 \mathrm{~m}^{2} / \mathrm{g}$, obtained by BET. 


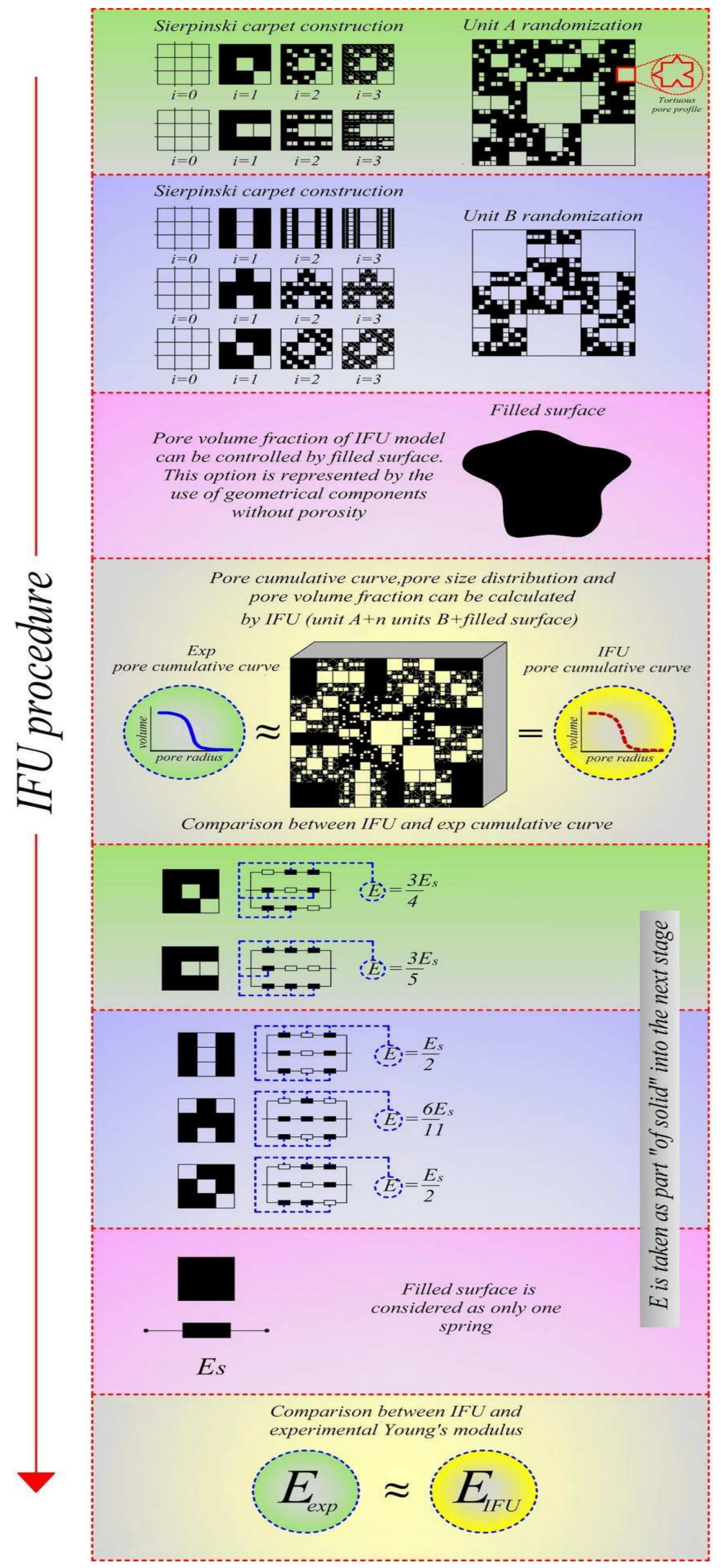

Figure 5. Representative scheme of IFU model working structure. 
Table 1. Input data of Intermingled Fractal Units' model.

\begin{tabular}{ccc}
\hline & & E1-1 \\
\hline & $\mathrm{D}_{\mathrm{f}}$ & 1.77 \\
& $\mathrm{n}$ & 1 \\
& $\mathrm{R}_{\max }(\mu \mathrm{m})$ & 16.5 \\
& Iteration & 4 \\
& $\mathrm{R}_{\min }(\mu \mathrm{m})$ & 0.2 \\
& Solid forever & 2 \\
\hline & $\mathrm{D}_{\mathrm{f}}$ & 1.89 \\
& $\mathrm{n}$ & 98 \\
& $\mathrm{R}_{\max }(\mu \mathrm{m})$ & 5.5 \\
& Iteration & 3 \\
& $\mathrm{R}_{\min }(\mu \mathrm{m})$ & 0.2 \\
& Solid forever & 4 \\
\hline & Filled surface $\left(\mu \mathrm{m}^{2}\right)$ & $1.12 \times 10^{5}$
\end{tabular}

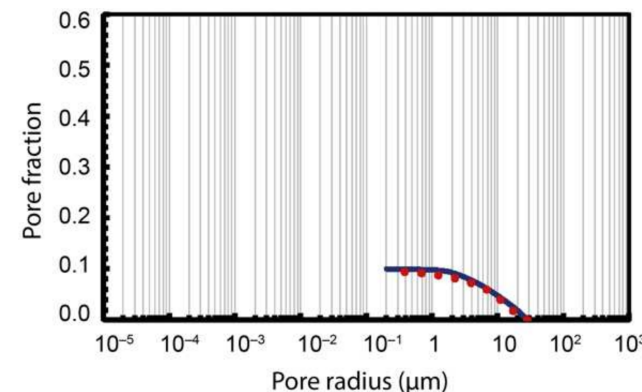

(a)

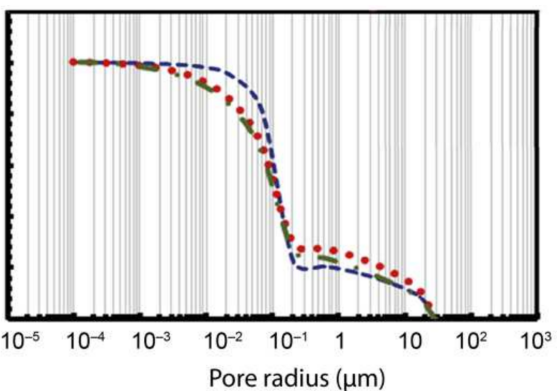

(b)

Figure 6. (a) Comparison between porosimetric curves obtained by IA and Fractal modeling; (b) model pore cumulative curves for typical NP Al, E1-1 (•), E1-2 (-), E1-3 (-), whose input data are reported in Table 2.

Table 2. Input data of fractal models for complete porous microstructure of NP Al.

\begin{tabular}{|c|c|c|c|c|}
\hline & & E1-1 & E1-2 & E1-3 \\
\hline \multirow{6}{*}{ Unit A } & $D_{f}$ & 1.89 & 1.89 & 1.77 \\
\hline & $\mathrm{n}$ & 1 & 1 & 1 \\
\hline & $\mathrm{R}_{\max }(\mu \mathrm{m})$ & 16.5 & 16.5 & 16.5 \\
\hline & Iteration & 10 & 10 & 10 \\
\hline & $\mathrm{R}_{\min }(\mu \mathrm{m})$ & 0.001 & 0.001 & 0.001 \\
\hline & Solid forever & 0 & 1 & 2 \\
\hline \multirow{7}{*}{ Unit B } & $D_{f}$ & 1.77 & 3 & 1.89 \\
\hline & $\mathrm{n}$ & 261 & 375 & 422 \\
\hline & $\mathrm{R}_{\max }(\mu \mathrm{m})$ & 1.83 & 1.83 & 1.83 \\
\hline & Iteration & 9 & 9 & 9 \\
\hline & $\mathrm{R}_{\min }(\mu \mathrm{m})$ & 0.2 & 0.2 & 0.2 \\
\hline & Solid forever & 5 & 0 & 2 \\
\hline & $\begin{array}{l}\text { Filled surface } \\
\qquad\left(\mu \mathrm{m}^{2}\right)\end{array}$ & 0 & 0 & 0 \\
\hline
\end{tabular}

The capability of the fractal model to describe porous structures allows using it for predicting elastic properties [25]. Based on Equation (10), the Young's modulus is estimated to be about $18 \pm 2 \mathrm{GPa}$. As mentioned above, the first experimental estimation of elastic behavior for NP Al is reported in this paper. Mechanical tests allow estimation of the value of the nanoindentation Young's modulus which is equal to $18.3 \pm 1.4 \mathrm{GPa}$, which is very close to that predicted by the model. 


\section{Conclusions}

We have shown that the VPD method can be successfully used to fabricate NP Al starting from an $\mathrm{Al}_{20} \mathrm{Zn}_{80}$ nanocomposite prepared by mechanical processing. Annealing the $\mathrm{Al}_{20} \mathrm{Zn}_{80}$ nanocomposite at $550{ }^{\circ} \mathrm{C}$ for $1 \mathrm{~h}$ allows removing $\mathrm{Zn}$ almost completely, leaving an NP Al structure with a high surface area. Moreover, using the SBF-SEM technique, a 3D reconstruction of the NP structure was obtained. A fractal model based on the Sierpinski carpet as a unit was proposed, and it showed the ability to model the porous microstructure of NP Al well. This phenomenological model allowed us to predict some features of this material, such as specific surface area and Young's elastic modulus, which are very close to experimental ones. The notable capability shown by this model to reproduce the experimental data encourages the development of this methodology which can in principle be applied to any possible NP metals.

The fabrication of NP Al with this method is unprecedented and can mark progress in the field. $\mathrm{Al}$ is a lightweight metal almost ubiquitous in modern technology and the availability of a lighter version of the metal can pave the way for a number of applications in strategic sectors of industry.

Supplementary Materials: The following are available online at https:/ /www.mdpi.com/article/10 .3390/app11146639/s1, Figure S1: EDS spectra, Figure S2: Examples of inverted SEM-SBF, Figure S3: Inverted and binarized SBF-SEM image, Figure S4: 3D reconstructions of NP Al, Video S1: Representative video of the porous structure evolution during the subsequent cuts of the entire cross-section profile.

Author Contributions: Conceptualization, A.P., G.P., F.D. and L.P.; investigation, A.P., M.F.C., E.S., A.F. and L.P.; modelling, G.P.; image processing and analysis, A.P., G.P., E.S. and A.F.; writingoriginal draft preparation, A.P., G.P., F.D., E.S., A.F. and L.P.; writing-review and editing, A.P., G.P., M.F.C., F.D., E.S., A.F. and L.P.; supervision, G.P. and L.P.; funding acquisition, G.P., A.F. All authors have read and agreed to the published version of the manuscript.

Funding: This research was funded by Fondazione di Sardegna, through projects F74I19001030007 and F72F20000360007. This research was also supported by King Abdullah University of Science (KAUST) Baseline funding to Andrea Falqui.

Institutional Review Board Statement: Not applicable.

Informed Consent Statement: Not applicable.

Data Availability Statement: The data presented in this study are included in this published article and in the supplementary material.

Acknowledgments: Fondazione di Sardegna and KAUST are gratefully acknowledged for supporting this research. One of the authors (A.P.) performed his activity in the framework of the International Ph.D. in Innovation Sciences and Technologies at the University of Cagliari, Italy. A.P. carried out part of his activity at the NABLA Lab, KAUST, which is gratefully acknowledged.

Conflicts of Interest: The authors declare no conflict of interest.

\section{References}

1. Ishizaki, K.; Komarneni, S.; Nanko, M. Introduction. In Porous Materials; Springer: Berlin/Heidelberg, Germany, 1998; pp. 1-11.

2. Ishizaki, K.; Komarneni, S.; Nanko, M. Properties and evaluation techniques for porous materials. In Porous Materials; Springer: Berlin/Heidelberg, Germany, 1998; pp. 202-224.

3. Banhart, J.; Weaire, D. On the road again: Metal foams find favor. Phys. Today 2002, 55, 37-42. [CrossRef]

4. Ding, Y.; Zhang, Z. Introduction to Nanoporous Metals. In Nanoporous Metals for Advanced Energy Technologies; Springer: Berlin/Heidelberg, Germany, 2016; pp. 1-35.

5. Banhart, J. Manufacture, characterisation and application of cellular metals and metal foams. Prog. Mater. Sci. 2001, 46, 559-632. [CrossRef]

6. Zhang, J.; Li, C.M. Nanoporous metals: Fabrication strategies and advanced electrochemical applications in catalysis, sensing and energy systems. Chem. Soc. Rev. 2012, 41, 7016-7031. [CrossRef] [PubMed] 
7. Fujita, T.; Qian, L.-H.H.; Inoke, K.; Erlebacher, J.; Chen, M.-W.W. Three-dimensional morphology of nanoporous gold. Appl. Phys. Lett. 2008, 92, 251902. [CrossRef]

8. Pia, G.; Delogu, F. Nanoporous Au: Statistical analysis of morphological features and evaluation of their influence on the elastic deformation behavior by phenomenological modeling. Acta Mater. 2015, 85, 250-260. [CrossRef]

9. Pia, G.; Carta, M.; Delogu, F. Nanoporous Au foams: Variation of effective Young's modulus with ligament size. Scr. Mater. 2018, 144, 22-26. [CrossRef]

10. Bryce, C.T.; Stephen, A.S.; Luther, E.P. Nanoporous metal foams. Angew. Chem. Int. Ed. 2010, 49, 4544-4565.

11. McCue, I.; Benn, E.; Gaskey, B.; Erlebacher, J. Dealloying and Dealloyed Materials. Annu. Rev. Mater. Res. 2016, 46, 263-286. [CrossRef]

12. Harrison, J.; Wagner, C. The attack of solid alloys by liquid metals and salt melts. Acta Metall. 1959, 7, 722-735. [CrossRef]

13. Wada, T.; Yubuta, K.; Inoue, A.; Kato, H. Dealloying by metallic melt. Mater. Lett. 2011, 65, 1076-1078. [CrossRef]

14. Guo, X.; Zhang, C.; Tian, Q.; Yu, D. Liquid metals dealloying as a general approach for the selective extraction of metals and the fabrication of nanoporous metals: A review. Mater. Today Commun. 2021, 26, 102007. [CrossRef]

15. Lu, Z.; Li, C.; Han, J.; Zhang, F.; Liu, P.; Wang, H.; Wang, Z.; Cheng, C.; Chen, L.; Hirata, A.; et al. Three-dimensional bicontinuous nanoporous materials by vapor phase dealloying. Nat. Commun. 2018, 9, 276. [CrossRef] [PubMed]

16. Corsi, J.S.; Fu, J.; Wang, Z.; Lee, T.; Ng, A.K.; Detsi, E. Hierarchical Bulk Nanoporous Aluminum for On-Site Generation of Hydrogen by Hydrolysis in Pure Water and Combustion of Solid Fuels. ACS Sustain. Chem. Eng. 2019, 7, 11194-11204. [CrossRef]

17. Garoli, D.; Schirato, A.; Giovannini, G.; Cattarin, S.; Ponzellini, P.; Calandrini, E.; Zaccaria, R.P.; D'amico, F.; Pachetti, M.; Yang, W.; et al. Galvanic replacement reaction as a route to prepare nanoporous aluminum for UV plasmonics. Nanomaterials 2020, 10, 102. [CrossRef] [PubMed]

18. Knight, M.W.; King, N.S.; Liu, L.; Everitt, H.O.; Nordlander, P.; Halas, N.J. Aluminum for Plasmonics. ACS Nano 2014, 8, 834-840. [CrossRef] [PubMed]

19. Mogensen, K.B.; Gühlke, M.; Kneipp, J.; Kadkhodazadeh, S.; Wagner, J.B.; Palanco, M.E.; Kneipp, H.; Kneipp, K. Surface-enhanced Raman scattering on aluminum using near infrared and visible excitation. Chem. Commun. 2014, 50, 3744-3746. [CrossRef]

20. Gopalakrishnan, M.; Sureshkumar, P.; Kanagarajan, V.; Thanusu, J. Aluminium metal powder (atomized) catalyzed Friedel-Crafts acylation in solvent-free conditions: A facile and rapid synthesis of aryl ketones under microwave irradiation. Catal. Commun. 2005, 6, 753-756. [CrossRef]

21. Witham, C.A.; Huang, W.; Tsung, C.K.; Kuhn, J.N.; Somorjai, G.A.; Toste, F.D. Converting homogeneous to heterogeneous in electrophilic catalysis using monodisperse metal nanoparticles. Nat. Chem. 2010, 2, 36-41. [CrossRef]

22. Huang, W.; Liu, J.H.C.; Alayoglu, P.; Li, Y.; Witham, C.A.; Tsung, C.K.; Toste, F.D.; Somorjai, G.A. Highly active heterogeneous palladium nanoparticle catalysts for homogeneous electrophilic reactions in solution and the utilization of a continuous flow reactor. J. Am. Chem. Soc. 2010, 132, 16771-16773. [CrossRef]

23. Suárez, O.M.; Estremera, E.G.; Soler, R.; Declet, A.; Hernández-Maldonado, A.J. Fabrication of Porous and Nanoporous Aluminum via Selective Dissolution of Al-Zn Alloys. Adv. Mater. Sci. Eng. 2014. [CrossRef]

24. Yang, W.; Zheng, X.-G.; Wang, S.-G.; Jin, H.-J. Nanoporous Aluminum by Galvanic Replacement: Dealloying and Inward-Growth Plating. J. Electrochem. Soc. 2018, 165, C492-C496. [CrossRef]

25. Casnedi, L.; Licheri, R.; Brun, M.; Pia, G. From nature geometry to material design: Advanced fractal nature analysis for predicting experimental elastic properties. Ceram. Int. 2020, 46, 23947-23955. [CrossRef]

26. Pia, G. High porous yttria-stabilized zirconia with aligned pore channels: Morphology directionality influence on heat transfer. Ceram. Int. 2016, 42, 11674-11681. [CrossRef]

27. Cai, J.; Perfect, E.; Cheng, C.L.; Hu, X. Generalized modeling of spontaneous imbibition based on hagen-poiseuille flow in tortuous capillaries with variably shaped apertures. Langmuir 2014, 30, 5142-5151. [CrossRef]

28. Rietveld, H.M. A profile refinement method for nuclear and magnetic structures. J. Appl. Crystallogr. 1969, 2, 65-71. [CrossRef]

29. Schindelin, J.; Arganda-Carreras, I.; Frise, E.; Kaynig, V.; Longair, M.; Pietzsch, T.; Preibisch, S.; Rueden, C.; Saalfeld, S.; Schmid, B.; et al. Fiji: An open-source platform for biological-image analysis. Nat. Methods 2012, 9, 676-682. [CrossRef]

30. Brunauer, S.; Emmett, P.H.; Teller, E. Adsorption of Gases in Multimolecular Layers. J. Am. Chem. Soc. 1938, 60, 309-319. [CrossRef]

31. Lubarda, V.A. On the effective lattice parameter of binary alloys. Mech. Mater. 2003, 35, 53-68. [CrossRef]

32. Skoko, Ž.; Štefani, G. Microstructure of Al-Zn and Zn-Al Alloys. Croat. Chem. Acta 2009, 82, 405-420.

33. Langhammer, C.; Schwind, M.; Kasemo, B.; Zorić, I. Localized surface plasmon resonances in aluminum nanodisks. Nano Lett. 2008, 8, 1461-1471. [CrossRef] [PubMed]

34. Thommes, M.; Kaneko, K.; Neimark, A.V.; Olivier, J.P.; Rodriguez-Reinoso, F.; Rouquerol, J.; Sing, K.S.W. Physisorption of gases, with special reference to the evaluation of surface area and pore size distribution (IUPAC Technical Report). Pure Appl. Chem. 2015, 87, 1051-1069. [CrossRef]

35. Lakshmanan, C.; Viswanath, R.N.; Polaki, S.R.; Rajaraman, R.; Dash, S.; Tyagi, A.K. Surface area of nanoporous gold: Effect on temperature. Electrochim. Acta 2015, 182, 565-572. [CrossRef]

36. Xu, H.; Pang, S.; Jin, Y.; Zhang, T. General synthesis of sponge-like ultrafine nanoporous metals by dealloying in citric acid. Nano Res. 2016, 9, 2467-2477. [CrossRef] 
37. Liu, Y.; Bliznakov, S.; Dimitrov, N. Comprehensive study of the application of a pb underpotential deposition-assisted method for surface area measurement of metallic nanoporous materials. J. Phys. Chem. C 2009, 113, 12362-12372. [CrossRef]

38. Ding, Y.; Erlebacher, J. Nanoporous metals with controlled multimodal pore size distribution. J. Am. Chem. Soc. 2003, 125, 7772-7773. [CrossRef] [PubMed]

39. Fujita, T. Hierarchical nanoporous metals as a path toward the ultimate three-dimensional functionality. Sci. Technol. Adv. Mater. 2017, 18, 724-740. [CrossRef] [PubMed] 